\title{
„Oser dépasser les frontières“ - Fronten aufbrechen im DaF-Unterricht durch kooperative Arbeit zwischen mehrsprachigen SchülerInnen und Studierenden im Oberelsass
}

\author{
Nina Kulovics \& Aline Vennemann
}

\begin{abstract}
Zusammenfassung
Dieser Bericht stellt ein mehrsprachiges Kunstprojekt im DaF-Unterricht zwischen SchülerInnen des Gymnasiums Jean-Henri Lambert und Studierenden der Universität Haute-Alsace in Mulhouse vor. Von Januar bis Mai 2015 wurde im Rahmen der Kooperation zwischen Sekundar- und Hochschule mit deutsch- und französischsprachigen Schriften aus dem Ersten Weltkrieg gearbeitet. Die zum Teil unveröffentlichten Texte stammen von Soldaten an der Westfront sowie von Zivilisten, die in der jeweiligen Muttersprache (Elsässisch; Deutsch; Französisch) von ihren Kriegserlebnissen in vielfältiger Weise berichten. Inwiefern eignen sich solche Zeitdokumente als Medien für einen nicht frontalen Fremdsprachenunterricht? Können sich Überlegungen zum Erlernen einer Fremdsprache und zur Landesgeschichte gegenseitig befruchten? Der performative Ansatz beim Einsatz historischen Materials im DaF-Unterricht stand im Mittelpunkt der Projektarbeit, die nicht nur geografische, also sichtbare Grenzen aufbrechen sollte, sondern auch mentale und soziale, d.h. verinnerlichte, unsichtbare Fronten lösen. Die vorliegende Untersuchung berücksichtigt nicht nur die Rahmenbedingungen des groß angelegten Projektunterrichts, sondern auch ursprüngliche Zielvorstellungen und konkrete Ergebnisse.
\end{abstract}

\section{1 ,(E)cri(t)s de guerre - Im Krieg schrei(b)en“: Projektvorstellung}

Ästhetische Erfahrungen wirken sich positiv auf das allgemeine Sprachbewusstsein und insbesondere auf das Fremdsprachenbewusstsein aus. Dies soll hier anhand eines fünfmonatigen DaF-Projekts gezeigt werden, das im Jahr 2015 in Mulhouse im Oberelsass durchgeführt wurde. SchülerInnen der gymnasialen Oberstufe und Studierende haben dort erstmals im Deutschunterricht innerhalb eines Kunstprojekts über den Ersten Weltkrieg zusammengearbeitet. 
Der interkulturelle Rahmen spielte dabei eine ganz besondere Rolle, insofern Lehrende und Lernende zum Teil elsässische, deutsche, schweizerische, österreichische und französische Wurzeln hatten und ihre sehr unterschiedlichen Sozialisierungshintergründe mit in den Fremdsprachenunterricht einbrachten. Deutsch als länderübergreifende Sprachvarietät wurde innerhalb eines Grenzen durchbrechenden und Konventionen aufhebenden Kunstprojekts produktiv eingesetzt, wobei Lehrende und Lernende sich differenziert auf empirischer und theoretischer Ebene mit unveröffentlichten Schriften von der Front im Ersten Weltkrieg auseinandergesetzt haben. Diese wurden sowohl musikalisch als auch sprachlich in Gegenwart der jeweiligen DaF-LehrerInnen ${ }^{1}$ sowie des lokalen Musikers Daniel Muringer ${ }^{2}$ für eine Performance im Mai 2015 aufbereitet. Das im Titel verankerte "Aufbrechen der Fronten" ${ }^{3}$ bezieht sich folglich nicht nur auf die behandelten Stoffe und die besondere Lokalisierung, sondern auch auf die pädagogisch-didaktische Ausrichtung des Projekts, das sich gezielt von frontalen Unterrichtsformen entfernt und Polaritäten kollektiv abzubauen, bzw. positiv aufzuladen sucht.

\section{2 „Oser dépasser les frontières“ - Fronten aufbrechen im DaF-Unterricht: Rahmenbedingungen und Zielsetzungen}

\subsection{Rahmenbedingungen}

Die Zielgruppe setzte sich aus Germanistikstudierenden im ersten Studienjahr und GymnasialschülerInnen einer $A b i B a c^{4}$-Klasse zusammen. Der projektorientierte, dem Autonomieprinzip Rechnung tragende Unterricht an der Hochschule wurde im Teamteaching an zwei bis drei Unterrichtseinheiten pro Woche durchgeführt. Das Sprachniveau lag nach den Kriterien des Gemeinsamen europäischen Referenzrahmens $(\mathrm{GeR})$ bei B1 bis C2. Als Zeitrahmen für die einzelnen Phasen zur Erarbeitung der dreisprachigen Aufführung im Mai 2015 wurden fünf Monate anberaumt.

\footnotetext{
${ }^{1}$ Der Bericht bezieht sich zwar auf unsere Erfahrung; allerdings waren weitere FachkollegInnen der Gymnasien Deck und Kastler in Guebwiller sowie eine Grundschule aus Saverne (Zabern) und Sélestat (Schlettstadt) an dem Projekt beteiligt. Am Tag der Aufführung waren aus logistischen Gründen leider nicht alle Schulen vertreten.

${ }^{2}$ Vgl. http://daniel.muringer.pagesperso-orange.fr/ und http://geranium-alsace. $\mathrm{com} /$ [zuletzt aufgerufen 31.03.2016].

${ }^{3}$ Dies ist nicht zuletzt auch das Motto von NovaTris, dem Zentrum für grenzüberschreitende Kompetenzen an der UHA Mulhouse, das unser Projekt unterstützt und subventioniert hat. Näheres siehe: http://www. novatris . uha.fr/ [zuletzt aufgerufen 31.03.2016].

${ }^{4}$ Seit 1994 kann an Schulen in Deutschland und Frankreich gleichzeitig das französische Baccalauréat und das deutsche Abitur erworben werden, vgl. http://www. france-allemagne. fr/AbiBac-Gleichzeitiger-Erwerb-der, 1433.html [zuletzt aufgerufen 31.03.2016].
} 


\subsection{Zielsetzungen}

Vorrangiges Ziel war das Aufbrechen von pädagogisch-didaktischen, institutionellen, kulturellen, sprachlichen, historischen und musikalischen Fronten. Oberste Priorität wurde dem selbst gesteuerten Lernen, der Teamarbeit in Projektgruppen und dem ästhetischen Lernen in einem dramapädagogischliterarisch-musikalisch orientiertem Setting eingeräumt. Durch handlungs- und projektorientierte Lehr- und Lernformen im kooperativen Teamteaching, das sich über strukturelle Fronten zwischen Schule und Universität hinweg setzte, wurden auch sprachliche Grenzen aufgebrochen.

Das Elsass ist eine mehrsprachige Region Frankreichs (vgl. Morgen 2004). Bei der Projektkonzeption wurde deshalb davon ausgegangen, dass standardsprachliches Deutsch eine plurizentrische Sprache ist und der Dialektvielfalt im alemannischen Dreiländereck auch im Deutschunterricht Respekt gezollt werden sollte.

Durch die spezielle Themenwahl wurde einerseits die historische Bedeutung des Elsass (nicht nur) im Ersten Weltkrieg zwischen deutschen und französischen „Fronten“ (vgl. Lienhard 2011) berücksichtigt, andrerseits die lokale Erinnerungskultur durch eine globale erweitert.

\section{Vom Klassenzimmer ins Audimax: Pädagogischer Aufbau, Arbeitsprozess und Ergebnisse}

\subsection{Pädagogischer Aufbau}

Das Projekt bestand aus drei Arbeitsphasen. Zuerst stellten Lehrende und Lernende mit Einverständnis des Musikers einen Text- und Liederkorpus zusammen, wobei nicht nur auf die Interessen der Beteiligten geachtet wurde, sondern auch auf den Bekanntheitsgrad und die Performativität der ausgewählten Schriften und Werke. Die musikalische Annäherung und Aneignung der größtenteils unveröffentlichten Materialien stand bei der endgültigen Auswahl im Vordergrund. Die Folgesitzungen widmeten sich der Text- und Spracharbeit, da es sich um eine Sammlung von Liedern, Gedichten, Fragmenten aus Memoiren und autobiographischen Schriften sowie freier Prosa aus der Zeit des Ersten Weltkrieges handelte, die in sowohl deutscher als auch französischer Sprache vorlagen. Die meisten wurden nämlich von elsässischen Soldaten an der Front ${ }^{5}$, SchriftstellerInnen, DichterInnen und Zeitzeugen verfasst, die von den traumatischen Kriegserlebnissen in ihrer Muttersprache berichten.

Eine intensive Auseinandersetzung mit jenen Zeitdokumenten setzte voraus, dass die Lernenden über genügend Kenntnisse über die Epoche und die AutorInnen verfügten. Neben der konzeptuellen und konkreten Aneignung der Werke mussten daher im Unterricht auch Entstehungsgeschichte und Kontext

\footnotetext{
${ }^{5}$ Auch hier tritt die Ambiguität des Begriffes Front zutage, insofern es sich aus deutscher Perspektive um die Westfront und französischer Perspektive um die Ostfront handelt.
} 
behandelt werden. Als besonders spannend erwiesen sich die unterschiedlichen Erfahrungshorizonte der SchülerInnen und Studierenden, die ja größtenteils aus dem Dreiländereck stammten und sich mit einer zum Teil unbekannten Heimatgeschichte befasst haben. Zu interkulturellen Missverständnissen konnte es durchaus kommen, als z.B. elsässische Texte in bilingualen Klassen durchgenommen wurden, die bisher keinen wirklichen Kontakt mit der Regionalsprache hatten. Vorurteile (auch der Eltern) mussten anfangs abgebaut werden, um dem Sprachunterricht, in dessen Rahmen das Projekt durchgeführt wurde, gerecht zu werden. Konkrete Arbeit mit Texten wie dem dreisprachigen Näsdla ${ }^{6}$-Dialog (vgl. Schittly 2013) wo es gleich zu Beginn in elsässischer Mundart heißt:

Ich kann nicht mit ihnen sprechen, man versteht sie ja nicht! Sie sprechen nicht wie wir! - Ja, hast du die Franzosen nicht gern? - Doch, ich hab' sie gern, besonders die Kavallerie, aber ich kann nicht mit ihnen sprechen ${ }^{7}$,

ermöglichten nur begrenzt, Hemmschwellen und Voreingenommenheit zu überwinden. Eine positive Erfahrung war hier das „Coming-Out“ einiger SchülerInnen und Studierender, die auf einmal ihre emotionale und linguistische Verbundenheit zur Regionalsprache zeigen und ausleben konnten.

Auf die eher mühsame Phase der Auseinandersetzung mit den Zeitdokumenten folgte die Etappe der Aneignung und Verkörperung. Um die Einzigartigkeit der Stoffe zu bewahren, mussten die Lernenden eine jeweils andere Form der Interpretation finden: Gesang, Theater, Rezitation oder Projektion. Dabei stand nicht das Endprodukt, sondern der Lernprozess im Mittelpunkt. SchülerInnen und Studierende sollten möglichst autonom arbeiten. Selbstinitiative und Teamgeist wurden insbesondere gefördert, gewohnte Rahmen und Rollenverteilungen aufgebrochen, so dass frontaler Unterricht notwendigerweise $\mathrm{zu}$ Lernbegleitung wurde. Die Lernenden mussten Titel und Inszenierungskonzepte selbständig entwickeln. Einzelne Arbeiten näherten sich sogar bestehenden Werken, wie z.B. das Projekt der „Todesharmonie“, wo die SchülerInnen des Lycée Lambert auf ähnliche Mittel zurückgriffen wie Einar Schleef in seiner berühmten Sportstück-Inszenierung am Burgtheater Wien im Jahre 1998.

Schließlich mag festgehalten werden, dass die Verlagerung des DaFUnterrichts vom Klassenzimmer ins Audimax sich als eine Erfahrung besonderen Werts herausstellte, die alle TeilnehmerInnen nicht nur zu einer breit gefächerten Gemeinschaft zusammenschloss, sondern auch den Orten, dem universitären Hörsaal, die ursprüngliche Funktion einer „maximalen Audienz“"zurück verlieh.

6 Teile des 1976 erschienenen Romans von Louis Schittly, einem 1938 geborenen dreisprachigen elsässischen Arzt, der Ärzte ohne Grenzen mitbegründete, können unter folgender Adresse angehört werden: http://lefuretmulhousien.fr/evenements/nasdla-ou-unautomne-sans-colchiques-lecture-publique-chez-bisey [zuletzt aufgerufen 31.03.2016].

7 Originalzitat: „Ech kà jo nét reeda médana; ma frschteet sa jo nét! Si reeda nét wia mér! Ja hàsch dü d'Franzoosa nét garn? - Doch! éch hà sa o garn; bsunders d'Kàvalrii; àwr' éch kà nét reeda médana." 
Nina Kulovics \& Aline Vennemann

„Oser dépasser les frontières“ - Fronten aufbrechen im DaF-Unterricht durch

kooperative Arbeit zwischen mehrsprachigen SchülerInnen und Studierenden

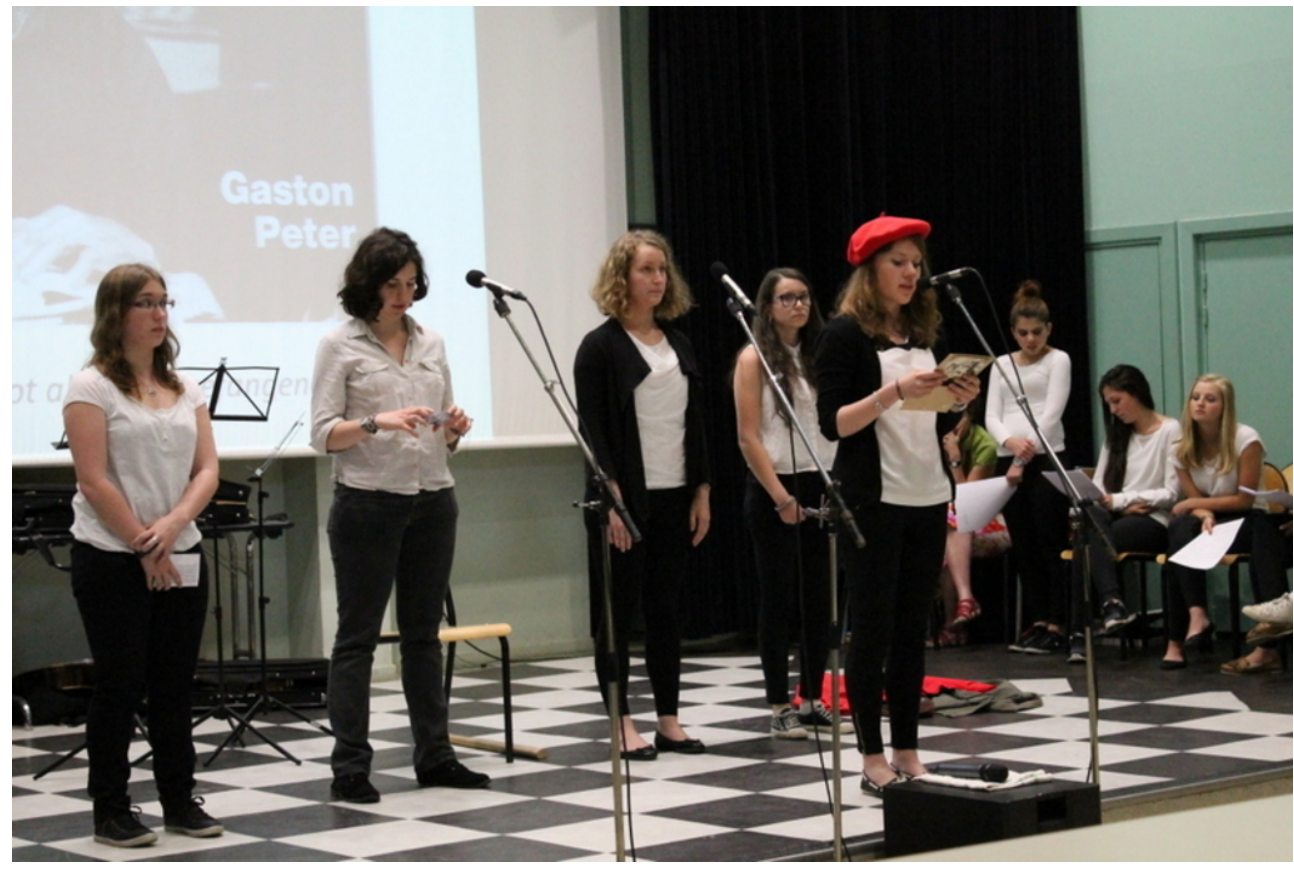

Abbildung 1: „Von der Vorlesung zur Performance“

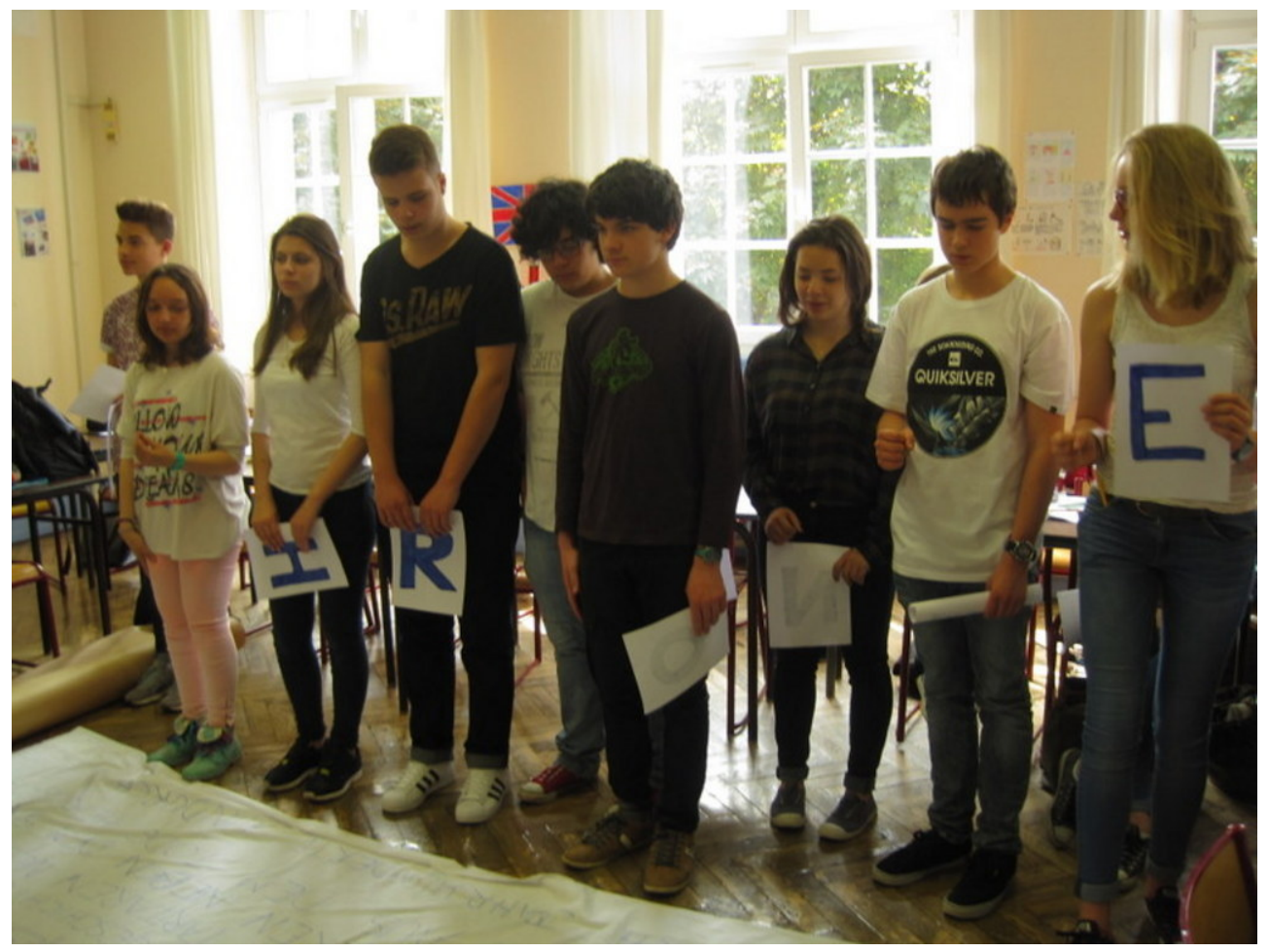

Abbildung 2: „Vom begrenzten Klassenraum ins Audimax“ 


\subsection{Arbeitsprozess und Ergebnisse}

In den unterschiedlichen Arbeitsphasen wurden nicht nur die Einbindung und Progression der SchülerInnen und Studierenden mitberücksichtigt, sondern auch - gemäß den neuen Lehrrichtlinien eines kompetenzorientierten Sprachunterrichts - den einzelnen Fähigkeiten aller TeilnehmerInnen volle Aufmerksamkeit geschenkt. Die unterschiedlichen Herangehensweisen sollten die Wahrnehmung der Spielenden sowie des Publikums abwechselnd anregen, wenn auch hauptsächlich auf der akustischen und visuellen Ebene gearbeitet wurde. Um die Autonomie der Lernenden zu stärken, haben die Projektleiterinnen die jeweiligen Aufgaben je nach Erfahrungshorizont und Neugierde der TeilnehmerInnen vergeben. Dies erforderte nicht nur auf der kommunikativen Ebene Teamgeist, Toleranz und Absprache, sondern auch reziproke Ehrlichkeit und Kompromissfähigkeit. Als besonders spannend hat sich dabei die Zusammenarbeit von Studierenden und SchülerInnen am Tag der Präsentationen herausgestellt.

Performative Prozesse fördern bekanntlich die Auseinandersetzung mit Sprache(n) in mündlicher und schriftlicher Form nachhaltiger als der traditionelle Frontalunterricht. Denn im interaktiven, kreativen und kooperativen prozessund produktionsorientierten Sprachunterricht wird die „vierte Wand“ durchbrochen und die künstlerische Seite der Lernenden und Lehrenden begünstigt. Ziel war u.a. ein spielerischer Einstieg in die ästhetische Als-ob-Realität, um die bei französischen SchülerInnen meist verdrängte Kreativität zu wecken. Somit versteht sich das DaF-Projekt als Alternative zu frontalen und linearen Unterrichtsformen. Die Ober- und HochschülerInnen hatten sichtlich Spaß an dieser neuen zweistimmigen und mehrsprachigen Unterrichtsgestaltung. Für die Projektleiterinnen war dies ein sichtbares Zeichen des erhofften Erfolgs.

Durch rhythmisches Sprechen, Stimm- und Sprechtraining (mit Rückgriff auf Übungen aus dem Gesangsunterricht und der Theaterpädagogik) unter Anleitung ihrer Lehrerinnen sowie des Musikers prägten sich die Lernenden die Texte selbständig ein. Ein Wegfall von Sprechhemmungen konnte zunehmend vermerkt werden. Außerdem wurde dadurch die Gruppendynamik gefördert.

Ein wesentlicher Teil des Projekts bestand in der grafischen Arbeit, die an die Hochschüler delegiert wurde (ohne die Ideen der SchülerInnen auszublenden): Es mussten Flyer ${ }^{8}$ entworfen werden, Werbetexte geschrieben und ein offizielles Plakat $^{9}$ angefertigt werden. Viel Kreativität zeigten die Studierenden bei der Titelfindung, denn es mussten ja beide Sprachen berücksichtigt werden. Nicht nur phonetische Aspekte spielten eine Rolle, sondern auch poetischliterarische sowie grafisch-praktische. Die Studierenden entschlossen sich für ein elliptisches Wortspiel, dass durch farblich gekennzeichnete Aussparung von

\footnotetext{
${ }^{8}$ Der Flyer wurde von den Germanistikstudierenden gestaltet und ist einsehbar unter: http: //www. novatris.uha.fr/wp-content/uploads/2015/03/BAT_Elyer_EcritsDeGuerre.pdf [zuletzt aufgerufen 31.03.2016].

9 Das Plakat wurde von den Germanistikstudierenden gestaltet und ist einsehbar unter: http://www.novatris.uha.fr/wp-content/uploads/2015/03/BAT_Affiche_ EcritsDeGuerre.jpg [zuletzt aufgerufen 31.03.2016].
} 
einzelnen Buchstaben zwei unterschiedliche Bedeutungen hervorbringt, die sowohl im Deutschen als auch im Französischen funktionieren.

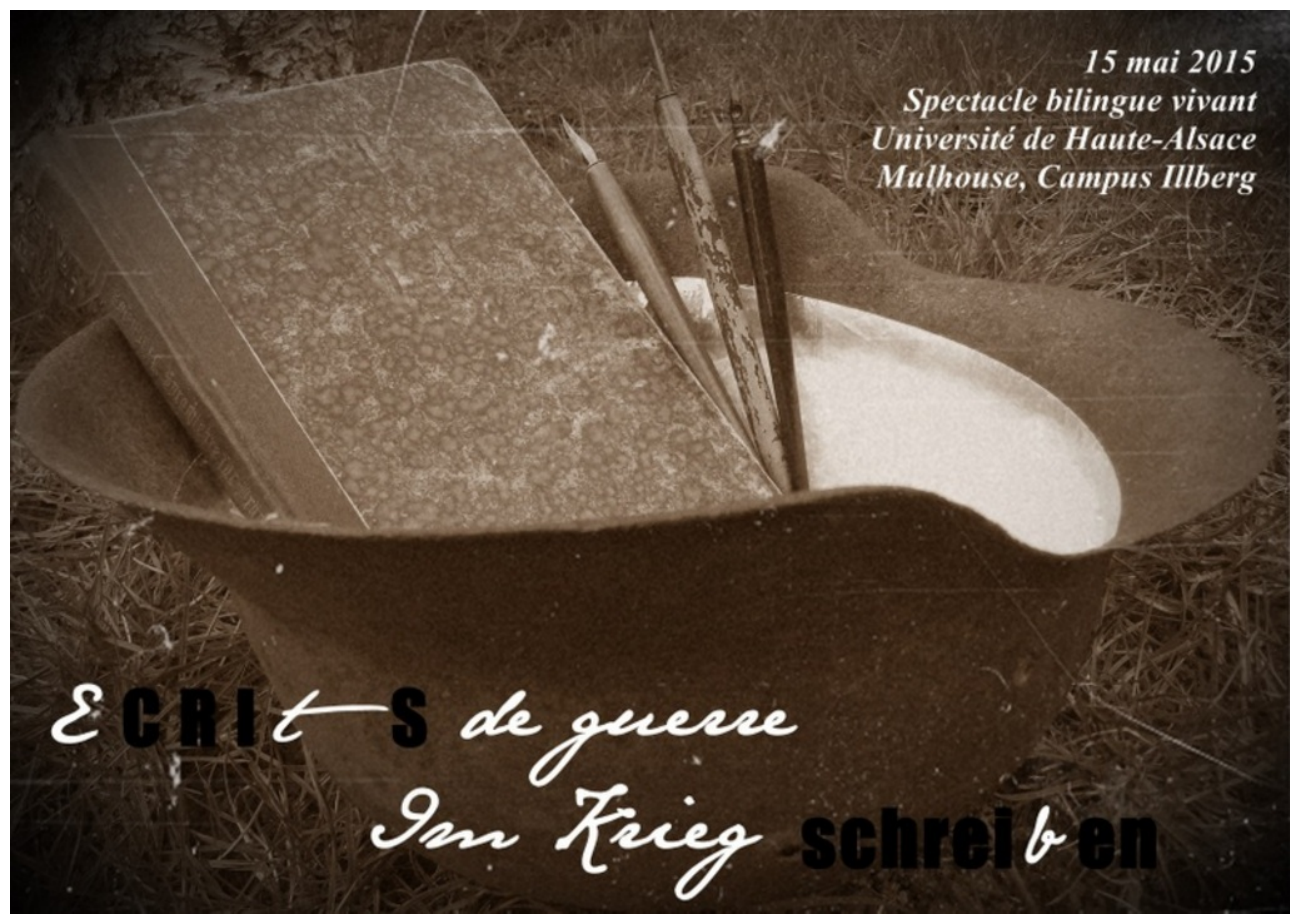

Abbildung 3: Flyer

Somit konnte auch stilistisches Grundwissen und dessen Anwendung in den Sprachunterricht eingebunden werden, ohne künstlich oder aufgesetzt zu wirken. Die kreative Gestaltung der Flyer und Plakate, des Layouts und der Drucksetzung sowie die gesamte Werbekampagne wurden von den Lernenden selbst durchgeführt und trugen zum selbstgesteuerten Lernen merklich bei. Sowohl für die SchülerInnen als auch für die Studierenden bedeutete diese Erfahrung eine grundlegende Ablösung von der gewohnten Unterrichtsform.

\section{Fazit und Ausblick}

Neben der historischen Einbettung und des regionalen Bezugs wurden durch dieses Kooperationsprojekt Fronten und Hemmschwellen in Bezug auf Institutionen, historische Gegebenheiten, Sprache(n), Unterrichtsmethoden, Interdisziplinarität und Altersunterschiede aufgehoben - denn auf der Bühne sind alle gleich und gemeinsam sind wir stark! Lehren und Lernen „mit Kopf, Herz, Hand und Fuß" (vgl. Schewe 1993: 44ff.) angereichert mit Teamgeist, Kreativität, Autonomie, Projektarbeit, Motivation, Offenheit, gegenseitigem Austausch und Performativität rückte den Lernprozess in all seinen Facetten in den Mittelpunkt.

Dank des bereichernden Projekts wurde nicht zuletzt von der Schul- und Universitätsleitung ein erhöhtes Interesse an Dramapädagogik im Fremd- 


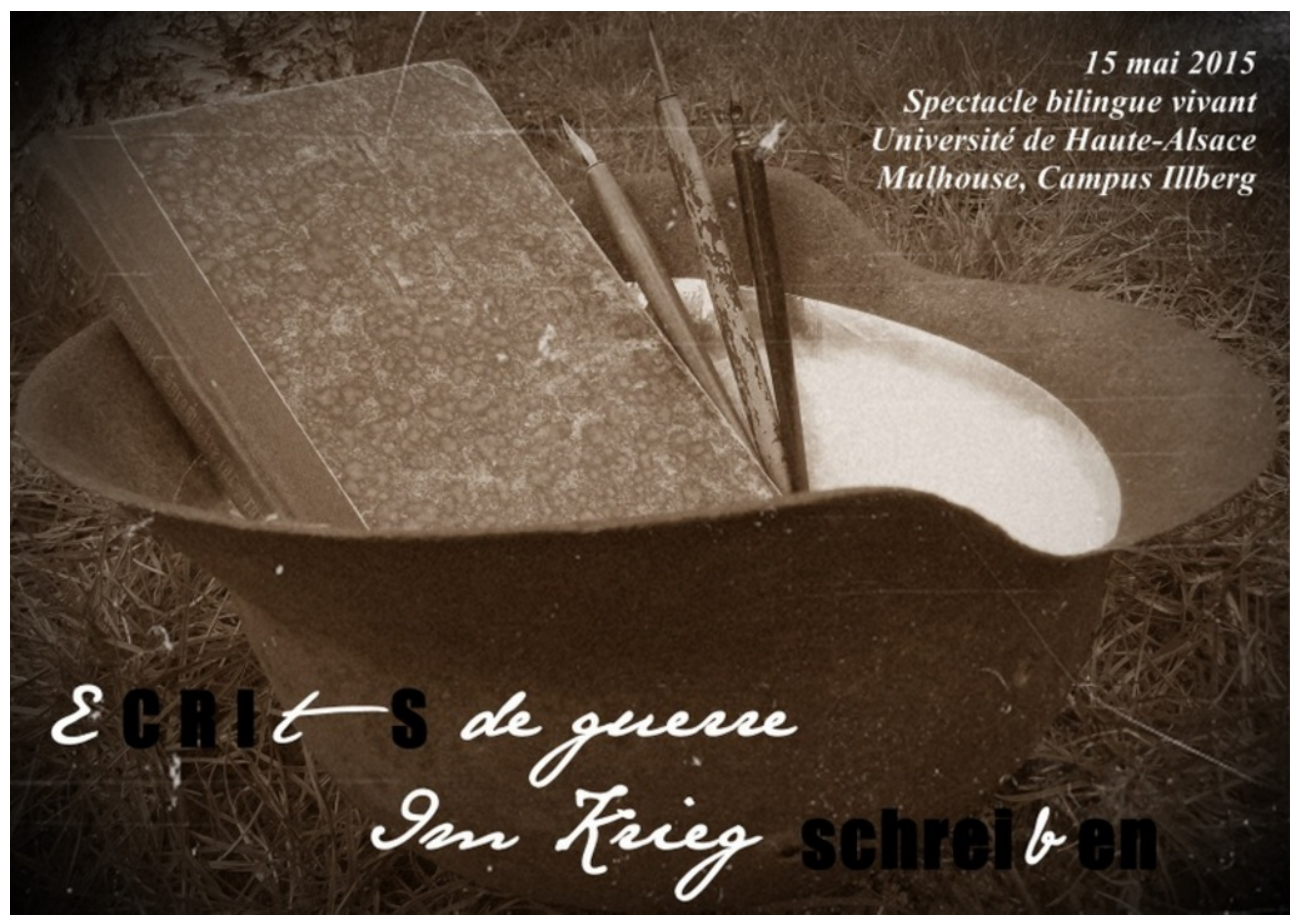

Abbildung 4: Flyer

sprachenunterricht bekundet. Auch die vielseitige Wirkung performativer Lernprozesse auf Deutschlernende sowie die Attraktivität für zukünftige Germanistikstudierende wurde hervorgehoben und das Potential performativer Lehr- und Lernformen positiv bewertet. Dies führte in weiterer Folge zu einem neuen Projekt im Studienjahr 2015/16 rund um das Leben des zweisprachigen elsässischen Arztes, Theologen, Musikers und Tierliebhabers Albert Schweitzer, wobei der TeilnehmerInnenkreis auf Anfrage der SchülerInnen und Studierenden ausgeweitet wurde. Am 4. Mai 2016 wurden die Ergebnisse in der Universitätsbibliothek erstmals von den GermanistInnen des 1., 2. und 3. Studienjahres sowie von den SchülerInnen der Seconde Abi-Bac und der Theatergruppe der Seconde 8 vorgestellt. ${ }^{10}$

\section{Bibliografie}

\section{AbiBac: http://www.france-allemagne.fr/AbiBac-Gleichzeitiger- Erwerb-der, 1433.html \\ [zuletzt aufgerufen 31.03.2016]}

\footnotetext{
10 Es handelt sich u.a. um zwei Theaterinszenierungen, eine deutsch-französische Schülerund Studierendenzeitung und eine aus den Arbeiten der Studierenden der UHA und der AbiBac-SchülerInnen des Lycée Lambert bestehenden Comicausstellung.
} 


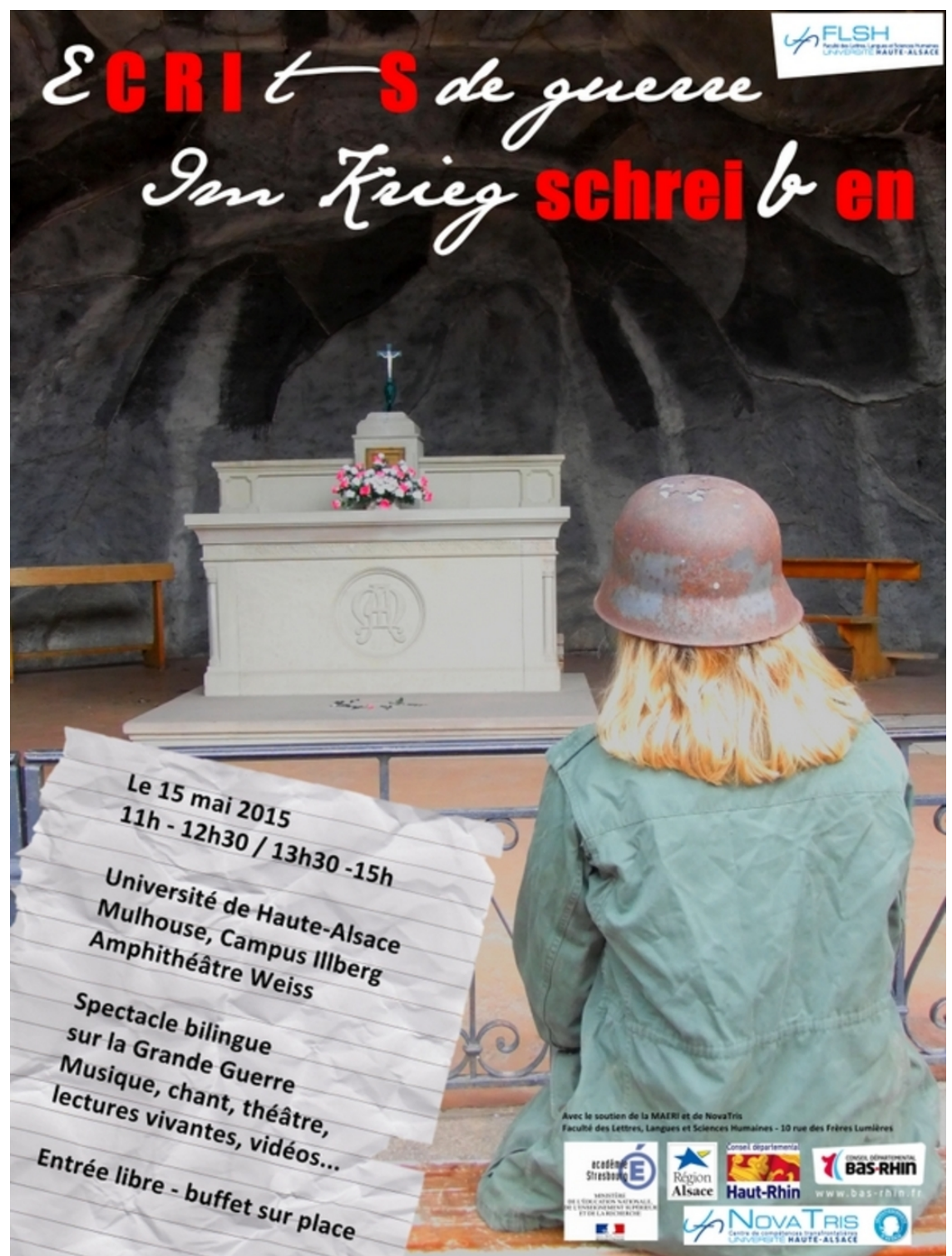

Abbildung 5: Plakat

Flyer zum Projekt „(E)cri(t)s de guerre - Im Krieg schrei(b)en“:

http://www.novatris.uha.fr/wp-

content/uploads/2015/03/BAT_Elyer_EcritsDeGuerre.pdf [zuletzt aufgerufen 31.03.2016]

Lienhard, Marc (2011): Histoire et Aléas de l'Identité Alsacienne. Strasbourg: La Nuée Bleue/DNA

Morgen, Daniel (2004): Die Sprachensituation im Elsass und das bilinguale Angebot in den Schulen im Elsass und am Oberrhein. In: Abel, Andrea et al. (Hrsg): Beiträge zum Thematischen Forum: «Mehrsprachigkeit in Grenzregionen» 3. Europäische Akademie Bozen (EURAC): 
http://www.eurac.edu/en/research/autonomies/commul/Documents /

LaBs/Morgen_final.pdf [zuletzt aufgerufen 31.03.2016]

Muringer, Daniel: http://daniel.muringer.pagesperso-orange.fr/ \& http://geranium-alsace.com/ [zuletzt aufgerufen 31.03.2016]

NovaTris, Centre de Compétences Transfrontalières, Université Haute-Alsace, Mulhouse: http://www.novatris . uha. fr/ [zuletzt aufgerufen 31.03.2016]

Plakat zum Projekt „(E)cri(t)s de guerre - Im Krieg schrei(b)en“:

http://www.novatris.uha.fr/wp-

content/uploads/2015/03/BAT_Affiche_EcritsDeGuerre.jpg [zuletzt aufgerufen 31.03.2016]

Schewe, Manfred (1993): Fremdsprache inszenieren: zur theoretischen Fundierung einer dramapädagogischen Lehr- und Lernpraxis. Oldenburg: Zentrum für Pädagogische Berufspraxis, Carl von Ossietzky Universität Oldenburg

Schittly, Louis (2013): Dr Näsdla ou Un autonme sans colchiques. Roman à lire à voix haute. Neuausgabe. Strasbourg: La Nuée Bleue/DNA

Schittly, Louis (n.d.): Öffentliche Lesung aus seinem Roman Dr Näsdla ou Un autonme sans colchiques in der Buchhandlung Bisey in Mulhouse und auf Radio MNE von Philippe Braun: https: / / youtu .be/OabI7018pps [zuletzt aufgerufen 31.03.2016] 